\title{
Study The Mechanism of Huangdi Anxiao Capsules In The Treatment of T2DM of Using UPLC-Q-TOF-MSE Combined With Network Pharmacological
}

\section{Wei Zhang ( $\square$ 2020205225015@stu.ahtcm.edu.cn )}

Anhui University of Traditional Chinese Medicine https://orcid.org/0000-0002-2305-0217

\section{Li Shan}

Anhui University of Traditional Chinese Medicine - East Campus: Anhui University of Traditional Chinese Medicine

\section{Meng Qing Zhu}

Anhui University of Traditional Chinese Medicine - Meishan Road Campus: Anhui University of Traditional Chinese Medicine

Zhao Hui Fang

Anhui University of Traditional Chinese Medicine - East Campus: Anhui University of Traditional Chinese Medicine

Xiao Chuang Liu

Anhui University of Traditional Chinese Medicine - East Campus: Anhui University of Traditional Chinese Medicine

Jia Rong Gao

Anhui University of Traditional Chinese Medicine https://orcid.org/0000-0002-8329-3106

\section{Research Article}

Keywords: Huangdi Anxiao capsule, Type 2 diabetes mellitus, UPLC-Q-TOF-MSE, Network pharmacology

Posted Date: January 3rd, 2022

DOI: https://doi.org/10.21203/rs.3.rs-1199441/v1

License: (1) This work is licensed under a Creative Commons Attribution 4.0 International License. Read Full License 


\section{Abstract}

This study was to explore the main material basis, target and pathway of Huangdi Anxiao capsule (HDAXC) for the treatment of type 2 diabetes mellitus (T2DM) by UPLC-Q-TOF-MSE and network pharmacology. In this study, HDAXC was administrated to T2DM rats, and its serum were detected by UPLC- Q-TOF-MS ${ }^{\mathrm{E}}$, and the prototype components of HDAXC were analyzed. Using Swiss target prediction database to predict the target of serum prototype components, using GeneCards and DrugBack database to predict the target of T2DM. These common targets are the prediction target of HDAXC acting on T2DM. The key components of HDAXC in the treatment of T2DM were determined by using the software of Cytoscape3.7.2 to visualize the results. Using the STRING online platform to construct the protein-protein interaction (PPI), the key target genes were selected. The Gene Ontology (GO) analysis and Kyoto Encyclopedia of Genes and Genomes (KEGG) pathway analysis of the common targets were carried out by using the OmicShare tools. And quantitative PCR and Western bolt were used to verify the related target genes. A toll of 28 prototype compounds were detected in rat serum, and 495 putative identified target genes were screened from HDAXC, of which 141 overlapped with the targets of T2DM and were considered potential therapeutic targets. The analysis of the network results showed that the key components of HDAXC are Magnoflorine, Galangin, Quercetin, and Epiberberine, etc. VEGFA, AKT1, SRC, EGFR might be the key target genes of HDAXC in the treatment of T2DM. HDAXC may have a therapeutic effect on T2DM by affecting HIF-1 signaling pathway, AGE-RAGE signaling pathway in diabetic complications, VEGFA signaling pathway and PI3K/AKT signaling pathways. In this study, compounds absorbed into the blood of HDAXC and its action target and pathway were preliminarily analyzed, which provided evidences for clarifying the chemical material basis and researching functional mechanism of HDAXC.

\section{Introduction}

Type 2 diabetes mellitus (T2DM) is a metabolic disorder caused by abnormal glucose and lipid metabolism caused by insufficient insulin secretion and insulin resistance and it will lead to the occurrence and development of a variety of complications with the continuation of the course of the disease (Belete. 2020). In 2019, it is estimated that $19.3 \%$ of people aged $65-99$ years live with diabetes. It is projected that the number of people older than 65 years (65-99 years) Xwith diabetes will reach 195.2 million by 2030 and 276.2 million by 2045 (Sinclair et al. 2020).

Traditional Chinese medicine (TCM) theories presented that the pathogenesis of T2DM (Chinese name: Xiaoke) lies in qi stagnation, blood stasis and phlegm retention leading to damp-heat accumulation in the stomach (Pan et al. 2020). Huangdi Anxiao Capsules (HDAXC) was created by the First Affiliated Hospital of Anhui University of Traditional Chinese Medicine and is used to treat traditional Chinese medicine preparations for T2DM. HDAXC consists of six herbs: Coptidis rhizoma (HL), Rehmanniae radix (SDH), Pueraria Lobate Radix (GG), Ophiopogonis radix (MD), Eriobotrya folium (PPY), Notoginseng radix et rhizome (SQ). It has been used clinically for many years and its efficacy is exactly (Gao et al. 2018). However, the pharmacodynamic substantial basis and mechanism of action of HDAXC are not clear, which affects its further development and application.

Ultra-performance liquid chromatography quadrupole time-of-flight mass spectrometry (UPLC-Q-TOF-MS ${ }^{\mathrm{E}}$ ) is a new analytical tool with good resolution, excellent sensitivity, and strong structural characterization capability (Huang et al. 2020). In recent years, UPLC-Q-TOF-MS ${ }^{\mathrm{E}}$ (where E represents collision energy) has provided a powerful approach for the efficient separation and structural characterization of TCM with the advantage of its high resolution, sensitivity and accuracy (Xu et al. 2020).

Based on systems biology, network pharmacology explores the correlation between drugs and diseases from an overall perspective. It has the characteristics of integrity and systematism, and is suitable for the efficacy mechanism and basic research of traditional Chinese medicine with multiple components, multiple targets and multiple pathways. A complex interaction network can be formed according to the target components, biological functions and bioactive compounds, etc., and the mechanism of action of TCM prescriptions can be elaborated from a systematic perspective. Network pharmacology is increasingly applied in Chinese medicine formula research (Luo et al. 2020). The schematic diagram of this study is shown in Figure 1.

\section{Materials And Methods}

\subsection{Chemicals, Materials and Reagents}

HPLC grade acetonitrile and methanol were urchased from Sigma-Aldrich (Shanghai) Trading Co., Ltd. (Shanghai, China). Formic acid was obtained from Shanghai Aladdin Biochemical Technology Co., Ltd. (Shanghai, China). Distilled water was purchased from Guangzhou Watsons Food \& Beverage Co., Ltd. (Guangzhou, China). The reference compounds Jatrorrhizine Hydrochloride, Quercetin and Notoginsenoside R1 were purchased from National Institutes for Food and Drug Control (Beijing, China). The reference compound Ononin was purchased from Shanghai Y ongheng Biotechnology Co., Ltd. (Shanghai, China). The reference compounds Ginsenoside Rg1, Ginsenoside Rb1, Coptisine, Epiberberine, Berberine hydrochloride and Palmatine were purchased from Chengdu Desite Biotechnology Co., Ltd. (Chengdu, China). The reference compounds Daidzin, Puerarin and Daidzein were purchased from Chengdu Must Biotechnology Co., Ltd. (Chengdu, China). The purity of each 
reference compound was determined to be over $98 \%$ by HPLC analysis. The Huangdi Anxiao Capsules were provided by the First Affiliated Hospital of Anhui University of traditional Chinese Medicine.

\subsection{Ethics Statement}

Spontaneous T2DM GK rats (200 \pm 20 g, 5 months old, SPF grade) were purchased from Shanghai Slack Laboratory Animals Co., Ltd., license number: SCXK (Shanghai) 2017-0005. All animals were raised in accordance with the guidelines for the care and use of laboratory animals and approved by the Animal Ethics Committee of the First Affiliated Hospital of Anhui University of Traditional Chinese Medicine.

\subsection{Preparation of HDAXC Solution and Reference Solution}

The accurately weighed $400 \mathrm{mg}$ the content of HDAXC was dispersed in 50ml methanol, ultrasonically in water bath (power $300 \mathrm{~W}$, frequency $40 \mathrm{KHZ}$ ) for $30 \mathrm{~min}$, filter it through a $0.22 \mu \mathrm{m}$ microporous filter membrane, and the filtrate was used for UPLC-Q-TOF-MS ${ }^{\mathrm{E}}$ analysis. 13 reference standards were dissolved in methanol. Before the analysis, they were mixed to make the concentration between $3 \sim 5 \mu \mathrm{g} / \mathrm{L}$ and filtered by $0.22 \mu \mathrm{m}$ 115 microporous filter membrane.

\subsection{Collection and Treatment of Serum Samples}

Random blood glucose of GK rats was higher than that of $11.1 \mathrm{mmol} / \mathrm{L}$ as the standard of successful T2DM model (Zhao et al. 2021). The T2DM GK rats were randomly divided into a GK control group $(n=5)$ and a GK experimental group $(n=5)$. The experimental group were given 15 $\mathrm{g} / \mathrm{kg}$ HDAXC twice a day for 3 days. 12 hours before the last intragastric administration, no feeding, free drinking water, and the last intragastric administration were given a full day dose. The control group was given $20 \mathrm{~mL} / \mathrm{kg}$ distilled water by gavage. Blood samples were collected an after oral 4 hours administration, which were allowed to stand at room temperature for $30 \mathrm{~min}$, centrifuged at 12,000 r/min for 5 min to obtain serum and store for use at $-20^{\circ} \mathrm{C}$. Taking $300 \mu \mathrm{l}$ serum sample in EP tube add $1200 \mu \mathrm{l}$ methanol to swirl for $30 \mathrm{~s}$, centrifuge for 5 min at 12000 $\mathrm{r} / \mathrm{min}$, taking out the supernatant, drying it with nitrogen (N2) at $40^{\circ} \mathrm{C}$, dissolving it again with $150 \mu \mathrm{l}$ methanol, swirling for $30 \mathrm{~s}$, centrifuging for $5 \mathrm{~min}$ at $12,000 \mathrm{r} / \mathrm{min}$, taking the supernatant was used for UPLC-Q-TOF-MS ${ }^{\mathrm{E}}$ analysis. Part for serum samples filtered through a $0.22 \mu \mathrm{m}$ filter membrane, heated at $56^{\circ} \mathrm{C}$ for $30 \mathrm{~min}$, and collected to store at $-80^{\circ} \mathrm{C}$. The serum samples were used for Quantitative PCR and Western bolt.

\subsection{Cell culture and treatment}

TNF-a-stimulated HUVEC cell model, which is often used for in vitro study of T2DM, has been mentioned in our previous study (Calandrelli R et al. 2020). Our experiment was separated into three groups: the control group, the model group, and the HDAXC treatment group. The cells were inoculated on a six-hole culture plate and cultured overnight. The storage solution of $20 \mathrm{~mol} / \mathrm{L}$ mannitol and $25 \mathrm{~mol} / \mathrm{L}$ glucose was configured respectively. The normal group added mannitol storage solution (culture medium itself contained $5 \mathrm{mM}$ glucose). The other groups were incubated in incubator for 48 hours after adding glucose storage solution and TNF-a to the final concentration of $25 \mathrm{mM}$ and $5 \mathrm{ng} / \mathrm{ml}$. In the drugcontaining group, $20 \%$ volume of drug-containing serum was added after 24 hours, and cultured in incubator for 24 hours. Then, the cells were collected for subsequent experiments.

\subsection{Chromatography and Mass Spectrometry Conditions}

Chromatographic analysis using a Waters Acquity UPLC system (Waters Corporation, Milford, USA). The column used for the experiment was

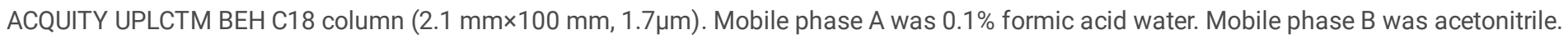
The gradient was set as follows: 0-1.5 min, 5\% B; 1.5-5 min, 5\% - 15\% B; 5-8 min, 15\% -20\% B; 8-9.5 min, 20\% B; 9.5-12 min, 20\% - 35\% B; 12-15.5 $\min , 35 \%-40 \%$ B; $15.5-17 \mathrm{~min}, 40 \%-50 \%$ B; $17-19 \mathrm{~min}, 50 \%-5 \% \mathrm{~B} ; 19-21 \mathrm{~min}, 5 \% \mathrm{~B}$. The column temperature was retained at $35{ }^{\circ} \mathrm{C}$ and the sample chamber temperature at $8{ }^{\circ} \mathrm{C}$. The flow rate was set at $0.2 \mathrm{~mL} / \mathrm{min}$. And sample injection volume was $1.0 \mu \mathrm{L}$.

The mass spectrometer used in the experiment was Waters Xevo G2 QTOF mass spectrometer (Waters Corporation, Milford, USA), and the Electrospray ionization source (ESI) included two modes, a negative ion mode and a positive ion mode. respectively, testing parameters: cone gas, $50 \mathrm{~L} / \mathrm{h}$; source temperature, $110^{\circ} \mathrm{C}(-)$ and $120^{\circ} \mathrm{C} \mathrm{(+)}$; desolvation gas temperature, $350{ }^{\circ} \mathrm{C}$; desolvation gas flow, $600 \mathrm{~L} / \mathrm{h}$; the capillary voltage is $2.5 \mathrm{kV}(\mathrm{ESI}+)$ in positive ion mode and $2.0 \mathrm{kV}(\mathrm{ESI})$ in negative ion mode; low collision energy, $6 \mathrm{~V}$; high collision energy, $20 \sim 40 \mathrm{~V}$; mass range $50 \sim 1200$.

\subsection{Network Pharmacology Analysis}

\subsubsection{Screening of Prototype Component Targets and T2DM Targets}

The prototype components identified by UPLC-Q-TOF-MS ${ }^{\mathrm{E}}$ were converted to simplified molecular input line entry specification (SMILES) in chem 2014 software. Inputing the conversion results into Swiss target prediction (http://www.swisstargetprediction.ch/), and set organization to "Homo sapiens" to search and filter the target of the components (Gfeller et al. 2014). 
In the GeneCards (http://www.genecards.org/) and DrugBack (https//www.drugbank.com/) enter the key word "type 2 diabetes mellitus", and search the corresponding target of the disease (Stelzer et al. 2016; Wishart et al. 2018). By combining prototype component targets with T2DM targets, the common targets of serum drug components and disease were selected. These common targets are the prediction target of HDAXC acting on T2DM.

\subsubsection{Construction and Analysis of "Component-Target" Network}

The prototype components and common targets are introduced into the software of Cytoscape3.7.2 to construct the component-target network. The network analyzer function in the software of Cytoscape3.7.2 is used to analyze the network. The node represents the absorbed prototype components and target of HDAXC. The edge shows the relationship between the components of TCM and targets. According to the results of topological analysis, the key compounds of HDAXC acting on T2DM were selected.

\subsubsection{The Protein-Protein Interaction Network Analysis of Common Targets}

The common targets were uploaded to STRING (https://string-db.org/) database, construct a protein-protein interaction (PPI) network, and set the protein type to "Homo sapiens", the minimum required interaction score is set to 0.400 , and the display hides the free point to obtain the PPI network. The data of PPI network was downloaded and imported into the software of Cytoscape3.7.2. The topological analysis of PPI network is carried out with the help of the "network analyzer" function of the software of Cytoscape3.7.2. The targets with degree of freedom, betweenness centrality and closeness centrality greater than the median are selected as the key targets.

\subsubsection{GO and KEGG Pathway Enrichment Analysis}

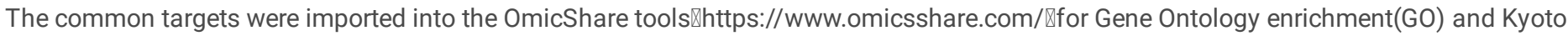
Encyclopedia of Genes and Genomes pathway analysis (KEGG)( Xiang L et al. 2021). The enrichment results of GO function and KEGG pathway were obtained by setting p-value. Go enrichment refers to the description of genes and proteins, mainly including three aspects: biological process (BP), molecular function (MF), and cellular component (CC).

\subsubsection{Construction of "Component-Target-Pathway" Network}

According to the enrichment results of KEGG pathway and literature search, the signal pathways related to T2DM were screened, the relevant targets of HDAXC in the treatment of T2DM were found, and the relevant information of HDAXC was combined to construct "component-targetpathway" network.

\subsection{In in-vivo experiments}

\subsubsection{Quantitative PCR Verifies the Effect of HDAXC on Gene Expression}

Total RNA from serum samples was isolated by TRIzol reagent (Thermo Fisher). Real-Time PCR System (PTC-200; Bio-Rad, Hercules, CA, USA). Removal of genomic DNA reaction: total RNA (mass $1 \mu \mathrm{g}$ ), $5 \times$ gDNA Eraser Buffer $2.0 \mu \mathrm{L}$ RNA Eraser $1.0 \mu \mathrm{L}$ were added into $0.2 \mathrm{~mL}$ tube, supplemented with DEPC water to $10 \mu \mathrm{L}$, gently mixed and centrifuged. PCR was heated at $42{ }^{\circ} \mathrm{C}$ for 2 min and immediately incubated with ice for 1 min. PrimeScript RT Enzyme Mix I $1.0 \mu$ L, RT Primer Mix $1.0 \mu$ L, RNase Free dH2O $4.0 \mu$ L and RevertAidTM M-MuLV Reverse Transcniptase $4.0 \mu \mathrm{L}$ are added to the EP tube. On the PCR instrument, $37^{\circ} \mathrm{C}, 15 \mathrm{~min}, 85^{\circ} \mathrm{C}, 5 \mathrm{~s}$. The above reaction solution was taken out and stored at $-20^{\circ} \mathrm{C}$, and quantitative PCR (qPCR) was performed using the ABI Real-Time PCR System (StepOne Plus; ABI). The primers are shown in Table 1. The relative expression of each target gene was quantified and normalized by $2-\Delta \Delta \mathrm{CT}$.

\subsubsection{Westem bolt Verifies the Effect of HDAXC on Gene Expression}

Each group included an equal mass of HUVEC cell samples and was subjected to western blot assay to detect the levels of AKT1, VEGFA, SRC, EGFR. Total proteins in HUVEC cell were lysed using RIPA lysis buffer. Lysates were centrifuged at 12,000 rpm for 10 min to obtain the supernatant, and the protein concentration was detected by ECL luminescence kit (UA276616, Thermo). Samples were mixed with the loading buffer and boiled for $10 \mathrm{~min}$ in a boiling water. Then, the protein was loaded on 10\% SDS-PAGE and transferred to PVDF membranes. Subsequently, the membranes were sealed with $5 \%$ skim milk powder at room temperature for $2 \mathrm{~h}$ with incubated primary antibody. Next, the membrane was washed with TBST and added to the secondary antibody to incubate at room temperature for $1 \mathrm{~h}$. Last, the color was developed after washing the membrane with TBST.

\subsection{Data Analysis}

Importing the mass spectrum data of drug-containing serum and blank serum into UNIFI software deduct the endogenous components in the serum, and compare the retention time, mass-to-charge ratio and secondary mass spectrum of the remaining ion peak with the ion peak of the HDAXC solution. If they are consistent, they are confirmed as the prototype components in HDAXC. SPSS20.0 software was used for data 
analysis and processing, and the results are expressed as mean \pm standard deviation (SD) ( $\overline{\mathrm{x}} \pm \mathrm{s})$. One-way analysis of variance (ANOVA) designed by randomized block design was used to analyze the differences in multiple groups. The result was considered statistically significant when $\mathrm{P}<0.05$.

\section{Results}

\subsection{Study on Serum Pharmacochemistry of HDAXC}

In the early stage of our research group, UPLC-Q-TOF-MSE technology has been applied to analyze the chemical components of HDAXC, and 100 chemical components have been analyzed from HDAXC (Gao et al. 2020). On this basis, this study analyzed the blood components of drugcontaining serum of HDAXC. The extracted ion chromatograms of the prototype components of drug-containing serum of HDAXC in positive and negative ion mode (Figure 2). A total of 28 prototype components were detected in drug-containing serum. The prototype components are mainly flavonoids, alkaloids and saponins, all of which analysis results (Table 2).

\subsection{Network Pharmacology Analysis}

\subsubsection{Target Prediction Results}

The 28 prototype components had a total of 1422 targets in the Swiss Target Prediction online database (Supplementary 1 ), and 495 targets were obtained after the duplicates were removed. Search through the GeneCards database and DrugBack database to obtain 1030 disease targets of T2DM and 782 targets were obtained after the duplicates were removed (Supplementary 2,3). After doing Venn diagram, 141 targets of T2DM and prototype components interaction were obtained, as shown in Figure 3.

\subsection{2 "Component-Target" Network construction results}

28 prototype components and 141 common targets were imported into the software of cytocsape3.7.2 to build a "component-target" interaction network, which has 181 nodes and 527 edges, as shown in Figure 4. Topology analysis of network relationship was carried out by using the network analyzer tool in cytocsape3.7.2. The 28 components are arranged in descending order according to Degree, Betweenness centrality and Closeness Centrality. The top five are Magnoflorine, Galangin, Quercetin and Epiberberine (Table 3). It is speculated that these compounds may be the key compounds of HDAXC in the treatment of T2DM.

\subsubsection{Construction and Analysis of Protein-Protein Interaction Network}

The above 141 targets are imported into the STRING database for PPI network analysis (Figure 5). The network includes 141 nodes and 1533 interaction relationships. The average degree of each node in the network is 16 . The average of Betweenness Centrality is 0.00291296 , and the average of Closeness Centrality is 0.46666667 . The results of STRING database are imported into Cytoscape3.7.2 for further analysis. Based on the three main parameters of degree, Betweenness Centrality and Closeness Centrality to centrality, targets above the median value are selected as key targets, and the median of degree is 16 , the median of Betweenness Centrality is 0.00291296 , and the median of Closeness Centrality is 0.46666667. There are 46 nodes whose degrees, Betweenness Centrality and Closeness Centrality exceed the average value (see Figure6, Table 4). It is speculated that these targets may be the key targets of HDAXC in the treatment of T2DM. The information regarding these targets are provided in Supplementary 4.

\subsubsection{Biological Function and Pathway Enrichment Analysis of Core Targets}

$\mathrm{GO}$ enrichment analysis was conducted on the above genes in Omicshare online software, and a total of 4953 genes were enriched. The results of GO enrichment analysis included Biological Process (BP), Molecular Function (MF) and Cell Component (CC). As shown in Figure 7, the top 30 in $B P, M F$ and $C C$ were screened from small to large according to $P$ values. Among them, BP enriched to 4170 items (7A), MF enriched to 467 items (7B), CC enriched to 316 items (7C). In order to further study how HDAXC affects T2DM through these target genes, the core target genes were uploaded to Omicshare online software and KEGG enrichment analysis was performed 201 related signaling pathways were obtained, including 139 important pathways, and the top 30 were selected according to $P$ values from small to large, as shown in figure 7D. KEGG pathway analysis of these genes revealed several pathways involved in the development and treatment of T2DM. The Pathways include HIF-1 signaling pathway (ko04066), AGE-RAGE signaling pathway in diabetic complications (ko04933), VEGF signaling pathway (ko04370) and PI3K-Akt signaling Pathway (KO04151), etc.

\subsection{5 "Component-Target-Pathway" Network construction results}

According to the enrichment results of KEGG pathway and literature search, 30 signal pathways closely related to T2DM were screened out, and the potential targets of HDAXC acting on T2DM in the pathway were identified. Prototype ingredients and targets intersected with T2DM and 
other information to build a network of "HDAXC-prescription composition-prototype components-targets-pathways", as shown in Figure 8, which intuitively reflects the process involved in the treatment of T2DM multiple active ingredients, targets and pathways.

\subsubsection{Effect of HDAXC on the mRNA and proteins expression of AKT1, VEGFA, SRC, and EGFR}

We chose four target genes with higher degrees to verify the influence of HDAXC. As shown in Figure 9 and Figure 10, compared with the control group, the expression levels of AKT1, VEGFA, SRC, and EGFR in the model group were significantly increased, while the expression levels of AKT1, VEGFA, SRC, and EGFR in the HDAXC group were significantly lower than those in the model group. The information regarding these targets are provided in Supplementary 5-7.

\section{Discussion}

Based on the method of serum pharmacochemistry and network pharmacology, with the help of UPLC-Q-TOF-MS ${ }^{\mathrm{E}}$ technology and corresponding database and software, this study systematically discussed the effective substances and mechanism of HDAXC in the treatment of T2DM by constructing "component-target" network, conducting the enrichment analysis of GO, KEGG pathway etc. Through the establishment of the "component-target" network of 28 prototype components of HDAXC, it was found that some compounds such as Magnoflorine, Galangin, Quercetin and Epiberberine played a key role in the treatment of T2DM. Magnoflorine has anti-inflammatory, antidiabetic, sedative and anxiolytic effects (Liang et al. 2020). It has been reported that Magnoflorine are found to be effective to control fasting blood glucose levels significantly in T2DM rats. It also promoted the Akt phosphorylation, suppressed autophagy and proteolysis (Yadav et al. 2021). Galangin attenuated cardiac oxidative injury, inflammation and apoptosis, and boosted antioxidant defenses Gal ameliorated hyperglycemia, dyslipidemia, and heart function markers, and prevented histopathological alterations in diabetic rats (Abukhalil et al. 2021). In vitro studies showed that galangin not only inhibits DPP-4 in a concentration-dependent manner but also regulates glucose levels, enabling the proliferation of rat L6 skeletal muscle cells (Kalhotra et al. 2019). In addition, it can reduce the level of glycogen induced in T2DM rats. The compound exerts potent anti-hyperglycemic effects by regulating the glucose homeostasis and reversing the glycolytic and gluconeogenic enzyme changes in rats (Aloud et al. 2020). Quercetin has anti-inflammatory and antidiabetic effects, animal studies have shown that quercetin can improve islet distortion in T2DM mice, especially mitochondrial atrophy and crest loss in pancreatic $\beta$ cells (Shuang et al. 2016; Li et al. 2020). In meanwhile, the quercetin prevented the development of oxidative stress in the T2DM rats cardiomyocytes by reducing NADPH oxidase and xanthine oxidase activities (Nataliia et al. 2021). Epiberberine has attracted considerable attention due to its anti-hyperglycemic, anti-hyperlipidemic, and anti-inflammatory functions (Xiao et al. 2021), in vitro showed that EPI inhibited the proliferation and induced the G2/M phase arrest of HG-induced GMCs and lower blood sugar to treat diabetic nephropathy. The above results shows that the therapeutic effect of HDAXC on T2DM is the result of multiple components. The results of PPI network analysis showed that the core targets of HDAXC in the treatment of T2DM were VEGFA, AKT1, SRC, MAPK3, EGFR, STAT3, CASP3, HRAS and ESR1 etc. these targets mainly involved in cell proliferation, inflammatory response and so on (Li et al. 2021; Seo et al. 2019; Xu et al. 2020; Sivaskandarajah et al. 2012; Xue et al. 2017).

According to research, Diabetes-related vascular diseases can lead to higher AKT1, VEGFA, SRC, EGFR levels in serum, while multiple reports say that genetic polymorphisms of these targets are closely related to insulin levels and large blood vessels. Zhao et al found that carvacrol administration significantly decreased the expression levels of AKT1 in db/db mice, suggested that carvacrol exerted protective effects on the liver in T2dM db/db mice (Zhao et al. 2021). Another study have shown that AKT1 is an important pathogenic target in T2DM. Lipopolysaccharide induced production of VEGF in whole blood cultures, and increases significantly in T2DM (Yin et al. 2020). It acts as a semiselective barrier and the structural integrity of vascular endothelium is essential for the maintenance of vascular homeostasis. Yang et al found that GSTpi stabilized VE-cadherin in endothelial cell membrane through inhibiting VE-cadherin phosphorylation and VE-cadherin/catenin complex dissociation in HUVECs, and consequently maintained endothelial barrier function (Yang et al. 2020). A recent study analyzed the genetic interaction between enhancers and protein-coding genes, suggesting that EGFR may be a new T2DM susceptibility gene (Yang et al. 2021).

GO analysis results show that the biological processes involved in Huangdi Anxiao Capsules acting on T2DM targets include transmembrane receptor protein tyrosine kinase signaling pathway(G0:0007169), positive regulation of phosphorylation(G0:0042327), cell proliferation(G0:0008283), cell death(G0:0008219) and so on; the molecular functions involved include enzyme binding(G0:0019899), protein kinase activity(G0:0004672) and so on; the cell composition involved receptor complex(G0:0043235), plasma membrane(G0:0005886).

The results of KEGG pathway enrichment show that Huangdi Anxiao capsule may play a therapeutic effect on type 2 diabetes by regulating HIF1 signaling pathway (ko04066), AGE-RAGE signaling pathway in diabetic complications(ko04933), VEGF signaling pathway(ko04370) and PI3KAkt signaling Pathway(ko04151). AGE/RAGE signaling has been shown to increase oxidative stress to promote diabetes-mediated vascular calcification through activation of Nox-1 and decreased expression of SOD-1 (Kay et al. 2016). LiuWei DiHuang Pill may play a role in the treatment of T2DM and its complications (atherosclerosis and nephropathy) through the AGE-RAGE signaling pathway, TNF signaling pathway, and NF-kappa B signaling pathway (He et al. 2019). In MG-treated HUVECs, glycine might inhibit the AGE/RAGE pathway and subsequent oxidative stress by improving Glo1 function, thus protecting against diabetic macrovascular complications (Wang et al. 2019). Curcumin played 
a role in diabetic cardiomyopathy treatment by modulating the Sirt1-Foxo1 and PI3K-Akt pathways (Ren et al. 2020). Activation of PI3K/ AKT/mTOR pathway and impaired autophagy can also lead to the occurrence of painful diabetic neuropathy (Liu et al. 2020). Adipose-derived stem cells can improve the neovascularization of diabetic ischemic skin by regulating the HIF-1a/VEGF pathway (Yu et al. 2018). High concentration of HIF-1a will stimulate the overexpression of vascular endothelial growth factor (VEGF), a downstream target gene (Shukla et al. 2017). VEGF can induce neovascularization and increase vascular permeability by regulating the proliferation and migration of vascular endothelial cells (Earle et al. 2019). The increase of VEGF level will promote the occurrence and development of diabetic angiopathy.

\section{Conclusion}

In conclusion, the mechanism of HDAXC in the treatment of T2DM involves a variety of active components, targets, and pathways. In this study, 28 active components, 46 potential targets, and 50 related signaling pathways in HDAXC were predicted. Among them, Magnoflorine, Galangin, Quercetin and Epiberberine and other components may act on AKT1, VEGFA, SRC, EGFR, and other targets, through HIF-1 signaling pathways, VEGF signaling pathways, AGE/RAGE signaling pathways, PI3K/AKT signaling pathways and participating in the inflammatory response in T2DM. This appears to be the mechanism underlying the therapeutic effect of HDAXC on T2DM, and it broadens the train of thought for followup pharmacological research.

\section{Declarations}

Author contribution Wei Zhang conceived and designed the study. performed some experiences and analyzed the data. Wei Zhang and Mengqing Zhu wrote the paper. Li Shan, Mengqing Zhu and Zhaohui Fang collected the information and did some experiments. Jia Rong Gao and Xiaochuang Liu reviewed and edited the manuscript. All authors read and approved the manuscript. The authors declare that all data were generated in-house and that no paper mill was used.

Funding This research was financially supported by the second batch of Scientific research projects of the national TCM clinical research base of the State Administration of traditional Chinese medicine (Grant No. JDZX2015126).

Data availability The data used and analyzed to support the findings of this study are available from the corresponding author upon request.

Ethics approval The animal protocol was approved by the Committee on the Ethics of Animal Experiments of Anhui University of Chinese medicine, and experimental procedures were carried out in accordance with their guidelines and regulations.

Conflict of interest The authors declare that they have no conflicts of interest.

\section{References}

1. Belete TM (2020) A Recent Achievement In the Discovery and Development of Novel Targets for the Treatment of Type-2 Diabetes Mellitus. Journal of experimental pharmacology 12:1-15. . doi. org/ 10.2147/JEP.S226113

2. Sinclair A, Saeedi P, Kaundal A et al (2020) Diabetes and global ageing among 65-99-year-old adults: Findings from the International Diabetes Federation Diabetes Atlas, 9th edition. Diabetes Research and Clinical Practice 162: 108078. doi. org/10.1016/j.diabres.2020.108078

3. Pan LL, Li ZZ, Wang YF et al (2020) Network pharmacology and metabolomics study on the intervention of traditional Chinese medicine Huanglian Decoction in rats with type 2 diabetes mellitus. J Ethnopharmacol 10258:112842. . doi. org/10.1016/j.jep.2020.112842

4. Gao JR, Guo MF, Fang ZH et al (2018) Effect of Huangdi Anxiao Capsules on zebrafish vascular lesions induced by high glucose and high fat. China J Chin Materia Med 43(21):4317-4322. . doi. org/10.19540/j.cnki.cjcmm.20180606.002

5. Huang ZZ, Du X, Ma CD et al (2020) Polygonatum sibiricumldentification of Antitumor Active Constituents in Flower by UPLC-Q-TOF-MS and Network Pharmacology. ACS omega 5(46):29755-29764. doi. org/10.1021/acsomega.0c03582

6. Xu L, Liu Y, Wu HF et al (2020) Rapid identification of chemical profile in Gandou decoction by UPLC-Q-TOF-MS coupled with novel informatics UNIFI platform. Journal of pharmaceutical analysis 10(1):35-48. . doi. org/10.1016/j.jpha.2019.05.003

7. Luo TT, Lu Y, Yan SK et al (2020) Network Pharmacology in Research of Chinese Medicine Formula: Methodology, Application and Prospective. Chin J Integr Med 26(1):72-80. . doi. org/10.1007/s11655-019-3064-0

8. Zhao JD, Li Y, Sun M et al (2021) Effect of berberine on hyperglycaemia and gut microbiota composition in type 2 diabetic Goto-Kakizaki rats. World J Gastroenterol 27(8):708-724. . doi. org/10.3748/wjg.v27.i8.708

9. Gfeller D, Grosdidier A, Wirth M et al (2014) SwissTargetPrediction: a web server for target prediction of bioactive small molecules. Nucleic acids research 42(Web Server issue):W32-38. . doi. org/10.1093/nar/gku293

10. Calandrelli R, Xu LX, Luo YJ et al (2020) Stress-induced RNA-chromatin interactions promote endothelial dysfunction. Nature communications, 2020, 11(1): 5211. https://doi.org/10.1038/s41467-020-18957-w

Page $7 / 23$ 
11. Stelzer G, Rosen N, Plaschkes I et al (2016) Current protocols in bioinformatics 54. . doi. org/ 10.1002/cpbi.5The GeneCards Suite: From Gene Data Mining to Disease Genome Sequence Analyses1.30.1-1.30.33

12. Wishart DS, Feunang YD, Guo AC et al (2018) DrugBank 5.0: a major update to the DrugBank database for 2018. Nucleic Acids Res 46(D1):D1074-D1082. . doi. org/ 10.1093/nar/gkx1037

13. Xiang L, Xing B, Liu X et al (2021) Network pharmacology-based research uncovers cold resistance and thermogenesis mechanism of Cinnamomum cassia. Fitoterapia 149:104824. https://doi. org/10.1016/j.fitote.2020.104824

14. Gao JR, Zhu MQ, Wang XL et al (2020) Identification of chemical constituents in Huangdi Anxiao Capsules by UPLC-Q-TOF-MS E combined with UNIFI software. China J Chin Materia Med 45(10):2395-2405. https://doi. org/10.19540/j.cnki.cjcmm.20191217.301

15. Liang XF, Xiang YN, Li YL et al (2020) A rapid method for simultaneous quantification of berberine, berbamine, magnoflorine and berberrubine in mouse serum using UPLC-MS/MS. Journal of chromatography B, Analytical technologies in the biomedical and life sciences 1142:122040. https://doi. org/10.1016/j.jchromb.2020.122040

16. Yadav A, Singh A, Phogat J et al (2021) Magnoflorine prevent the skeletal muscle atrophy via Akt/mTOR/FoxO signal pathway and increase slow-MyHC production in streptozotocin-induced diabetic rats. J Ethnopharmacol 267:113510. https://doi. org/10.1016/j.jep.2020.113510

17. Abukhalil MH, Althunibat OY, Aladaileh SH et al (2021) Galangin attenuates diabetic cardiomyopathy through modulating oxidative stress, inflammation and apoptosis in rats. Biomedecine \& pharmacotherapie 138:111410... org/ 10.1016/j.biopha.2021.111410

18. Kalhotra P, Chittepu VCSR, Revilla GO et al (2019) Discovery of Galangin as a Potential DPP-4 Inhibitor That Improves Insulin-Stimulated Skeletal Muscle Glucose Uptake: A Combinational Therapy for Diabetes. Int J Mol Sci 20(5):1228. https://doi.org/ 10.3390/ijms20051228

19. Aloud AA, Chinnadurai V, Chandramohan G et al (2020) Galangin controls streptozotocin-caused glucose homeostasis and reverses glycolytic and gluconeogenic enzyme changes in rats. Arch Physiol Biochem 126(2):101-106. https://doi.org/ 10.1080/13813455.2018.1498521

20. Shuang C, Jiang HM, Wu XS et al (2016) Therapeutic Effects of Quercetin on Inflammation, Obesity, and Type 2 Diabetes. Mediat Inflamm 2016:9340637. https://doi.org/ 10.1155/2016/9340637

21. Li D, Jiang CJ, Mei GB et al (2020) Quercetin Alleviates Ferroptosis of Pancreatic $\beta$ Cells in Type 2 Diabetes. Nutrients 12(10):2954. https://doi.org/10.3390/nu12102954

22. Nataliia IG, Borikov OY, Kiprych TV et al (2021) Quercetin improves myocardial redox status in rats with type 2 diabetes. Endocr Regul 55(3):142-152. https://doi.org/10.2478/enr-2021-0015

23. Xiao YP, Deng JL, Li CM et al (2021) Epiberberine ameliorated diabetic nephropathy by inactivating the angiotensinogen (Agt) to repress TGF $\beta$ /Smad2 pathway. Phytomedicine: international journal of phytotherapy and phytopharmacology 83:153488. https://doi.org/10.1016/j.phymed.2021.153488

24. Li Y, Pan Y, Cao SR et al (2021) Podocyte EGFR Inhibits Autophagy Through Upregulation of Rubicon in Type 2 Diabetic Nephropathy. Diabetes 70(2):562-576. https://doi.org/ 10.2337/db20-0660

25. Seo J, Guk G, Park SH et al (2019) Tyrosine phosphorylation of HDAC3 by Src kinase mediates proliferation of HER2-positive breast cancer cells. J Cell Physiol 234(5):6428-6436. https://doi.org/10.1002/jcp.27378

26. Xu Y, Jiang W, Zhong LL et al (2020) miR-195-5p alleviates acute kidney injury through repression of inflammation and oxidative stress by targeting vascular endothelial growth factor A 12(11): 10235-10245. https://doi.org/ 10.18632/aging.103160

27. Sivaskandarajah GA, Jeansson M, Maezawa $Y$ et al (2012) Vegfa protects the glomerular microvasculature in diabetes. Diabetes 61(11):2958-2966. . doi: 10.2337/db11-1655

28. Xue JF, Shi ZM, Zou J et al (2017) Inhibition of PI3K/AKT/mTOR signaling pathway promotes autophagy of articular chondrocytes and attenuates inflammatory response in rats with osteoarthritis. Biomed Pharmacother 89:1252-1261. https://doi.org/ 10.1016/j.biopha.2017.01.130

29. Zhao W, Chen L, Zhou H et al (2021) Protective effect of carvacrol on liver injury in type 2 diabetic db/db mice. Mol Med Rep $24(5): 741$. https://doi.org/ 10.3892/mmr.2021.12381

30. Yin B, Bi YM, Fan GJ et al (2020) Molecular Mechanism of the Effect of Huanglian Jiedu Decoction on Type 2 Diabetes Mellitus Based on Network Pharmacology and Molecular Docking. J Diabetes Res 2020:5273914. https://doi.org/10.1155/2020/5273914

31. Yang Y, Dong XL, Zheng SN et al (2020) GSTpi regulates VE-cadherin stabilization through promoting S-glutathionylation of Src. Redox Biol 30:101416. https://doi.org/ 10.1016/j.redox.2019.101416

32. Yang Y, Yao S, Ding JM et al (2021) Enhancer-Gene Interaction Analyses Identified the Epidermal Growth Factor Receptor as a Susceptibility Gene for Type 2 Diabetes Mellitus. Diabetes \& metabolism journal 45(2):241-250. https://doi.org/10.4093/dmj.2019.0204

33. Kay AM, Simpson CL, Stewart JA (2016) The Role of AGE/RAGE Signaling in Diabetes-Mediated Vascular Calcification. J Diabetes Res 2016:6809703. https://doi.org/ 10.1155/2016/6809703

Page $8 / 23$ 
34. He D, Huang JH, Zhang ZY et al (2019) A Network Pharmacology-Based Strategy For Predicting Active Ingredients And Potential Targets Of LiuWei DiHuang Pill In Treating Type 2 Diabetes Mellitus. Drug design. development and therapy 13:3989-4005. https://doi.org/ 10.2147/DDDT.S216644

35. Wang ZW, Zhang JQ, Chen L et al (2019) Glycine Suppresses AGE/RAGE Signaling Pathway and Subsequent Oxidative Stress by Restoring Glo1 Function in the Aorta of Diabetic Rats and in HUVECs. Oxidative medicine and cellular longevity, 2019:4628962. https://doi.org/ $10.1155 / 2019 / 4628962$

36. Ren BC, Zhang YF, Liu SS et al (2020) Curcumin alleviates oxidative stress and inhibits apoptosis in diabetic cardiomyopathy via Sirt1Foxo1 and PI3K-Akt signalling pathways. J Cell Mol Med 24(21):12355-12367. https://doi.org/ 10.1111/jcmm.15725

37. Liu K, Yang YC, Zhou F et al (2020) Inhibition of PI3K/AKT/mTOR signaling pathway promotes autophagy and relieves hyperalgesia in diabetic rats. NeuroReport 31(9):644-649. https://doi.org/ 10.1097/WNR.0000000000001461

38. Yu WY, Sun W et al (2018) Adipose-derived stem cells improve neovascularization in ischemic flaps in diabetic mellitus through HIF1a/VEGF pathway. Eur Rev Med Pharmacol Sci 22(1):10-16. https://doi.org/10.26355/eurrev_201801_14094

39. Shukla R, Pandey N, Banerjee S et al (2017) Effect of extract of Pueraria tuberosa on expression of hypoxia inducible factor-1a and vascular endothelial growth factor in kidney of diabetic rats. Biomed Pharmacother 93:276-285. https://doi.org/10.1016/j.biopha.2017.06.045

40. Earle KA, Zitouni K, Zadeh JN (2019) Lipopolysaccharide-Induced VEGF Production and Ambient Oxidative Stress in Type 2 Diabetes. J Clin Endocrinol Metab 104(1):1-6. https://doi.org/10.1210/jc.2018-00836

\section{Tables}

Table 1 Information of primers for RT-qPCR

\begin{tabular}{|c|c|c|}
\hline Targeted gene & Forward sequence and reverse sequence & Amplicon Size \\
\hline \multirow[t]{2}{*}{$\beta$-actin } & F:5'-CCCTGGAGAAGAGCTACGAG-3' & \multirow[t]{2}{*}{96} \\
\hline & R:5'-GGAAGGAAGGCTGGAAGAGT-3' & \\
\hline \multirow[t]{2}{*}{ AKT1 } & F:5'-CTTTCGGCAAGGTGATCCTG-3' & \multirow[t]{2}{*}{167} \\
\hline & R:5'-GTACTTCAGGGCTGTGAGGA-3' & \\
\hline \multirow[t]{2}{*}{ VEGFA } & F:5'-TTTGGGAACACCGACAAACC-3' & \multirow[t]{2}{*}{97} \\
\hline & R:5'-GGTGTCCTCATCCCTGTACC-3' & \\
\hline \multirow[t]{2}{*}{ SRC } & F:5'-CTGCTCAATGCAGAGAACCC-3' & \multirow[t]{2}{*}{87} \\
\hline & R:5'-GTCAGACACTGAGAGGCAGT-3' & \\
\hline \multirow[t]{2}{*}{ EGFR } & F:5'-CAGATCGCAAAGGGCATGAA-3' & \multirow[t]{2}{*}{167} \\
\hline & R:5'-TTGCCTCCTTCTGCATGGTA-3' & \\
\hline
\end{tabular}

Table 2 Identification of prototype components in rat serum of Huangdi Anxiao Capsules by UPLC-Q-TOF-MS 


\begin{tabular}{|c|c|c|c|c|c|c|c|c|c|c|}
\hline \multirow[t]{2}{*}{ No. } & \multirow[t]{2}{*}{$\operatorname{Rt}(\min )$} & \multirow{2}{*}{$\begin{array}{l}\text { Identified } \\
\text { compounds }\end{array}$} & \multicolumn{2}{|c|}{ Positive ion(m/z) } & \multicolumn{2}{|c|}{ Negative ion(m/z) } & \multirow[t]{2}{*}{ Formula } & \multirow[t]{2}{*}{$\operatorname{Mv}(D a)$} & \multirow[t]{2}{*}{$\mathrm{MS} / \mathrm{MS}(\mathrm{m} / \mathrm{z})$} & \multirow[t]{2}{*}{ Source } \\
\hline & & & indicated & ppm & indicated & ppm & & & & \\
\hline 1 & 3.08 & Phenylalanine & - & - & 164.0712 & -3.1 & C9H11NO2 & 165.0783 & 164,147 & SDH1 \\
\hline \multirow[t]{2}{*}{2} & \multirow[t]{2}{*}{5.55} & \multirow[t]{2}{*}{$\begin{array}{l}\text { Genistein-8-C- } \\
\text { glucoside }\end{array}$} & \multirow[t]{2}{*}{433.1131} & \multirow[t]{2}{*}{0.4} & \multirow[t]{2}{*}{431.0989} & \multirow[t]{2}{*}{1.2} & \multirow[t]{2}{*}{$\mathrm{C} 21 \mathrm{H} 20010$} & \multirow[t]{2}{*}{432.1058} & $\begin{array}{l}433,415,397 \\
313,431,413\end{array}$ & \multirow[t]{2}{*}{ GG1 } \\
\hline & & & & & & & & & $395,311,152$ & \\
\hline \multirow[t]{2}{*}{3} & \multirow[t]{2}{*}{6.41} & \multirow[t]{2}{*}{ Puerarin* } & \multirow[t]{2}{*}{417.117} & \multirow[t]{2}{*}{3.8} & \multirow[t]{2}{*}{415.1066} & \multirow[t]{2}{*}{8.9} & \multirow[t]{2}{*}{ C21H20O9 } & \multirow[t]{2}{*}{416.1078} & $\begin{array}{l}417,399,381 \\
363,351\end{array}$ & GG2 \\
\hline & & & & & & & & & $\begin{array}{l}321,297,279 \\
415,295,267\end{array}$ & \\
\hline 4 & 6.6 & Puerarinxyloside & 549.16 & -0.5 & 547.1436 & -3.8 & $\mathrm{C} 26 \mathrm{H} 28013$ & 548.1509 & $\begin{array}{l}549,399,381 \\
363,297,255\end{array}$ & GG3 \\
\hline & & & & & & & & & $547,415,253$ & \\
\hline 5 & 6.74 & 5-Hydroxyononin & - & - & 445.1124 & -3.5 & $\mathrm{C} 22 \mathrm{H} 22010$ & 446.1197 & 445,283 & GG4 \\
\hline 6 & 6.81 & RehMapicroside & - & - & 345.154 & -4.3 & $\mathrm{C} 16 \mathrm{H} 26 \mathrm{O} 8$ & 346.1613 & 345,183 & $\mathrm{SDH} 2$ \\
\hline 7 & 6.96 & Magnoflorine & 342.1712 & 4.6 & - & - & $\mathrm{C} 2 \mathrm{OH} 24 \mathrm{NO} 4+$ & 342.1712 & $\begin{array}{l}342,297,265 \\
237\end{array}$ & HL1 \\
\hline 8 & 7.33 & Daidzin* & 417.1165 & -3.6 & 461.1109 & 4.2 & C21H20O9 & 416.1104 & $\begin{array}{l}417,255,415 \\
253\end{array}$ & GG5 \\
\hline 9 & 9.46 & Berberrubine & 322.1097 & 0.7 & - & - & $\mathrm{C} 19 \mathrm{H} 16 \mathrm{NO} 4+$ & 322.1097 & $322,306,278$ & HL2 \\
\hline 10 & 11.47 & Columbamine & 338.1405 & 3.8 & - & - & $\mathrm{C} 2 \mathrm{OH} 2 \mathrm{ONO} 4+$ & 338.1405 & $338,322,294$ & HL3 \\
\hline & & & & & & & & & $280,262,234$ & \\
\hline 11 & 11.6 & Epiberberine* & 336.1226 & -2.9 & - & - & C20H18NO4+ & 336.1229 & $\begin{array}{l}336,320,306, \\
292,278\end{array}$ & HL4 \\
\hline 12 & 11.67 & Coptisine* & 320.0929 & 1.8 & - & - & $\mathrm{C} 19 \mathrm{H} 14 \mathrm{NO4+}$ & 320.0929 & $\begin{array}{l}320,292,262, \\
234\end{array}$ & HL5 \\
\hline 13 & 11.74 & Scoparone & - & - & 251.0561 & -0.1 & C11H1004 & 206.0579 & $251,191,161$ & GG9 \\
\hline 14 & 11.77 & Jatrorrizine* & 338.1405 & 3.8 & - & - & $\mathrm{C} 2 \mathrm{OH} 2 \mathrm{ONO} 4+$ & 338.1405 & 338,294 & HL6 \\
\hline & & & & & & & & & $280,262,234$ & \\
\hline 15 & 12.2 & $\begin{array}{l}\text { Notoginsenoside } \\
\text { R1* }\end{array}$ & - & - & 977.5295 & -2.9 & C47H80018 & 932.5316 & $\begin{array}{l}977,931,799 \\
637,475\end{array}$ & SQ2 \\
\hline 16 & 12.52 & Ginsenoside Rg1* & - & - & 845.4876 & -3.3 & $\mathrm{C} 42 \mathrm{H} 72 \mathrm{O} 14$ & 800.4894 & $\begin{array}{l}845,799,637 \\
619,475\end{array}$ & SQ3 \\
\hline 17 & 12.52 & Ononin* & 431.1337 & 0.2 & 475.1236 & -2.1 & $\mathrm{C} 22 \mathrm{H} 22 \mathrm{O} 9$ & 430.1254 & $\begin{array}{l}431,269,255 \\
475,267,253\end{array}$ & GG6 \\
\hline 18 & 12.66 & $\begin{array}{l}\text { Ophiopogonanone } \\
\text { F }\end{array}$ & - & - & 419.1356 & 1.9 & $\mathrm{C} 2 \mathrm{OH} 22 \mathrm{O}$ & 374.1374 & 419,343 & MD1 \\
\hline 19 & 12.66 & Daidzein* & 255.0648 & -1.5 & 253.0495 & -4.6 & $\mathrm{C} 15 \mathrm{H} 1004$ & 254.0567 & $\begin{array}{l}255,237,137 \\
119,253,135\end{array}$ & GG5 \\
\hline & & & & & & & & & 117 & \\
\hline 20 & 12.88 & Palmatine* & 352.1565 & 4.5 & - & - & $\mathrm{C} 21 \mathrm{H} 22 \mathrm{NO} 4+$ & 352.1565 & $\begin{array}{l}352,336,308 \\
292,264\end{array}$ & HL7 \\
\hline 21 & 12.95 & $\begin{array}{l}\text { 3'- } \\
\text { Methoxydaidzein }\end{array}$ & 285.0761 & 1.3 & 283.0612 & -0.1 & $\mathrm{C} 16 \mathrm{H} 12 \mathrm{O} 5$ & 284.0699 & $\begin{array}{l}285,271,255 \\
137,283,268\end{array}$ & GG8 \\
\hline & & & & & & & & & 253 & \\
\hline 22 & 13.02 & Berberine* & 336.1266 & 8.9 & - & - & $\mathrm{C} 2 \mathrm{OH} 18 \mathrm{NO} 4+$ & 336.1266 & $\begin{array}{l}336,320,306, \\
292,278\end{array}$ & HL8 \\
\hline 23 & 13.25 & Quercetin* & - & - & 301.0366 & 3.9 & C15H1007 & 302.0438 & 301,151 & SQ1 \\
\hline
\end{tabular}




\begin{tabular}{|c|c|c|c|c|c|c|c|c|c|c|}
\hline 24 & 14.14 & Galangin & 271.0597 & -1.5 & 269.0451 & 0.3 & $\mathrm{C} 15 \mathrm{H} 1005$ & 270.0524 & $\begin{array}{l}271,163,153 \\
119,269,161\end{array}$ & PPY1 \\
\hline \multirow[t]{2}{*}{25} & 14.27 & Ginsenoside Rb1* & 1109.6112 & 0.8 & 1153.5996 & -1.3 & C54H92O23 & 1108.6014 & $\begin{array}{l}1109,947 \\
785,623 \\
1153,1107\end{array}$ & SQ4 \\
\hline & & & & & & & & & $\begin{array}{l}945,783,621 \\
553,459\end{array}$ & \\
\hline \multirow[t]{2}{*}{26} & 14.27 & Ginsenoside Rk1 & 767.4934 & -0.8 & - & - & $\mathrm{C} 42 \mathrm{H} 70012$ & 766.4861 & $\begin{array}{l}767,605,443 \\
425,407,343\end{array}$ & SQ5 \\
\hline & & & & & & & & & 325 & \\
\hline 27 & 16.15 & $\begin{array}{l}\text { Notoginsenoside } \\
\mathrm{K}\end{array}$ & - & - & 991.5489 & 0.6 & C48H82O18 & 946.5501 & $\begin{array}{l}991,783,621, \\
603,537\end{array}$ & SQ6 \\
\hline 28 & 16.15 & Lupenone & 425.3766 & -2.8 & - & - & $\mathrm{C} 30 \mathrm{H} 480$ & 424.3693 & $425,409+$ & GG10 \\
\hline
\end{tabular}

Note: *: indicates the components confirmed by comparison with the reference standards; -: indicates no detection; SDH: Rehmanniae Radix; HL: Coptis Rhizoma; GG: Pueraria Lobate Radix; PPY: Eriobotryae Folium; MD: Ophiopogonis Radix; SQ: Notoginseng Radix Et Rhizoma

Table 3 Basic information of key compounds in the treatment of T2DM with HDAXC 


\begin{tabular}{|c|c|c|c|c|}
\hline name & Compound & Degree & Betweenness Centrality & Closeness Centrality \\
\hline HL1 & Magnoflorine & 47 & 0.253963 & 0.423529 \\
\hline PPY1 & Galangin & 46 & 0.147851 & 0.421546 \\
\hline SQ1 & Quercetin & 45 & 0.142322 & 0.417633 \\
\hline HL4 & Epiberberine & 35 & 0.116463 & 0.399113 \\
\hline HL6 & Jatrorrizine & 31 & 0.080305 & 0.390456 \\
\hline HL8 & Berberine & 31 & 0.075102 & 0.387097 \\
\hline HL7 & Palmatine & 29 & 0.082388 & 0.375783 \\
\hline MD1 & OphiopogonanoneF & 28 & 0.095449 & 0.385439 \\
\hline GG7 & Daidzein & 27 & 0.066652 & 0.387097 \\
\hline GG8 & 3'-Methoxydaidzein & 27 & 0.065354 & 0.387097 \\
\hline HL3 & Columbamine & 24 & 0.047231 & 0.356436 \\
\hline GG6 & Ononin & 16 & 0.040172 & 0.357853 \\
\hline GG10 & Lupenone & 14 & 0.046757 & 0.359281 \\
\hline GG2 & Puerarin & 13 & 0.029998 & 0.360721 \\
\hline $\mathrm{SDH} 2$ & RehMapicroside & 12 & 0.054745 & 0.340265 \\
\hline SDH1 & Phenylalanine & 12 & 0.02251 & 0.345489 \\
\hline HL2 & Berberrubine & 12 & 0.006979 & 0.344168 \\
\hline GG4 & 5-Hydroxyononin & 10 & 0.015265 & 0.337711 \\
\hline GG5 & Daidzin & 10 & 0.006558 & 0.35225 \\
\hline GG1 & Genistein-8-C-glucoside & 9 & 0.007962 & 0.346821 \\
\hline GG9 & Scoparone & 9 & 0.002407 & 0.309811 \\
\hline HL5 & Coptisine & 9 & 0.00148 & 0.316344 \\
\hline SQ2 & NotoginsenosideR1 & 8 & 0.022554 & 0.35225 \\
\hline SQ4 & 5-Hydroxyononin & 7 & 0.007753 & 0.279938 \\
\hline SQ3 & GinsenosideRg1 & 5 & 0.00648 & 0.301508 \\
\hline SQ6 & NotoginsenosideK & 4 & 0.001192 & 0.246914 \\
\hline SQ5 & GinsenosideRk1 & 4 & 0.001192 & 0.246914 \\
\hline GG3 & Puerarinxyloside & 3 & 0.000342 & 0.32316 \\
\hline
\end{tabular}

Table 4 Basic information of key target of HDAXC in the treatment of T2DM 


\begin{tabular}{|c|c|c|c|c|c|c|c|}
\hline name & Degree & BetweennessCentrality & ClosenessCentrality & name & Degree & BetweennessCentrality & ClosenessCentrality \\
\hline TNF & 75 & 0.0677996 & 0.648148 & ICAM1 & 38 & 0.004102 & 0.520446 \\
\hline VEGFA & 75 & 0.0451885 & 0.651163 & APP & 37 & 0.033006 & 0.546875 \\
\hline AKT1 & 73 & 0.0498529 & 0.654206 & JAK2 & 37 & 0.018001 & 0.524345 \\
\hline SRC & 67 & 0.0520175 & 0.636364 & PPARA & 36 & 0.028383 & 0.532319 \\
\hline MAPK3 & 65 & 0.0432191 & 0.625 & MET & 35 & 0.003622 & 0.518519 \\
\hline EGFR & 65 & 0.0275212 & 0.630631 & FGFR1 & 31 & 0.020476 & 0.507246 \\
\hline STAT3 & 62 & 0.0155258 & 0.59322 & PDGFRB & 31 & 0.003298 & 0.507246 \\
\hline CASP3 & 61 & 0.030528 & 0.619469 & $\mathrm{~F} 2$ & 29 & 0.026189 & 0.524345 \\
\hline HRAS & 60 & 0.0204615 & 0.59322 & NTRK1 & 29 & 0.005557 & 0.512821 \\
\hline ESR1 & 59 & 0.0235368 & 0.595745 & PTPN1 & 29 & 0.003234 & 0.507246 \\
\hline PPARG & 58 & 0.0538791 & 0.585774 & PRKCB & 27 & 0.048888 & 0.48951 \\
\hline ERBB2 & 56 & 0.0127201 & 0.578512 & FLT1 & 27 & 0.004766 & 0.487805 \\
\hline MMP9 & 55 & 0.0267689 & 0.566802 & RAF1 & 27 & 0.003002 & 0.501792 \\
\hline FGF2 & 55 & 0.0195778 & 0.580913 & ABCB1 & 26 & 0.014515 & 0.509091 \\
\hline PTGS2 & 53 & 0.0177557 & 0.580913 & $\mathrm{TH}$ & 25 & 0.029371 & 0.516605 \\
\hline MTOR & 53 & 0.0107443 & 0.580913 & ABCG2 & 25 & 0.019993 & 0.5 \\
\hline MAPK1 & 50 & 0.0083613 & 0.571429 & CYP19A1 & 24 & 0.020494 & 0.498221 \\
\hline KDR & 47 & 0.0152082 & 0.557769 & MPO & 24 & 0.004719 & 0.4947 \\
\hline MMP2 & 44 & 0.0053956 & 0.538462 & CYP2D6 & 21 & 0.025695 & 0.47619 \\
\hline IL2 & 40 & 0.0050083 & 0.530303 & AHR & 21 & 0.006593 & 0.505415 \\
\hline MAPK14 & 39 & 0.0149247 & 0.540541 & AKR1B1 & 19 & 0.020753 & 0.4947 \\
\hline AR & 38 & 0.0108846 & 0.530303 & ADRB2 & 18 & 0.023585 & 0.503597 \\
\hline SIRT1 & 38 & 0.0053238 & 0.520446 & CYP1A1 & 16 & 0.005692 & 0.479452 \\
\hline
\end{tabular}

\section{Figures}



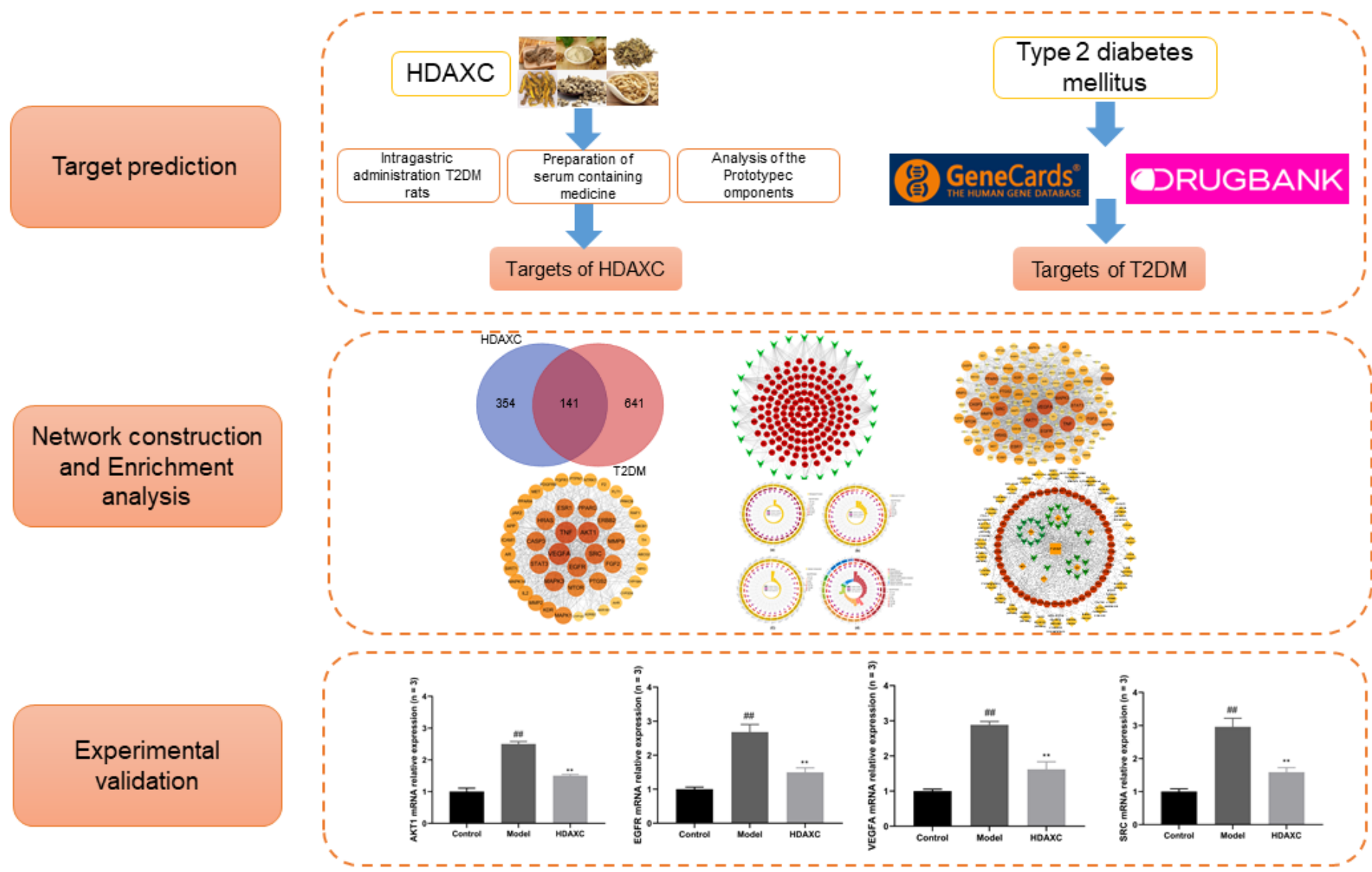

Figure 1

The whole framework based on an integration strategy of network pharmacology 

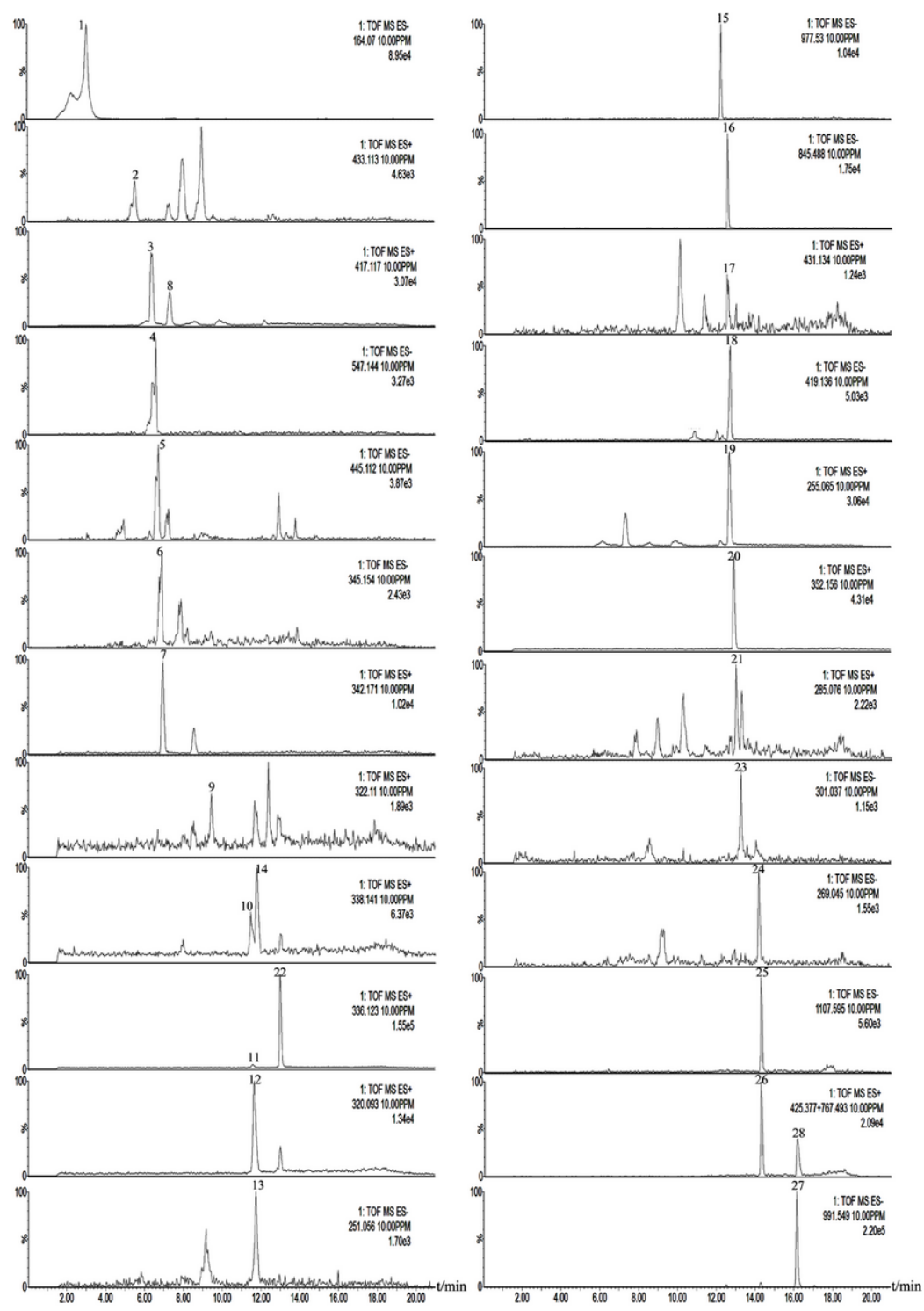

Figure 2

The extracted ion chromatograms (EICs) of the prototype components of HDAXC-dosed rats serum in positive and negative ion mode 


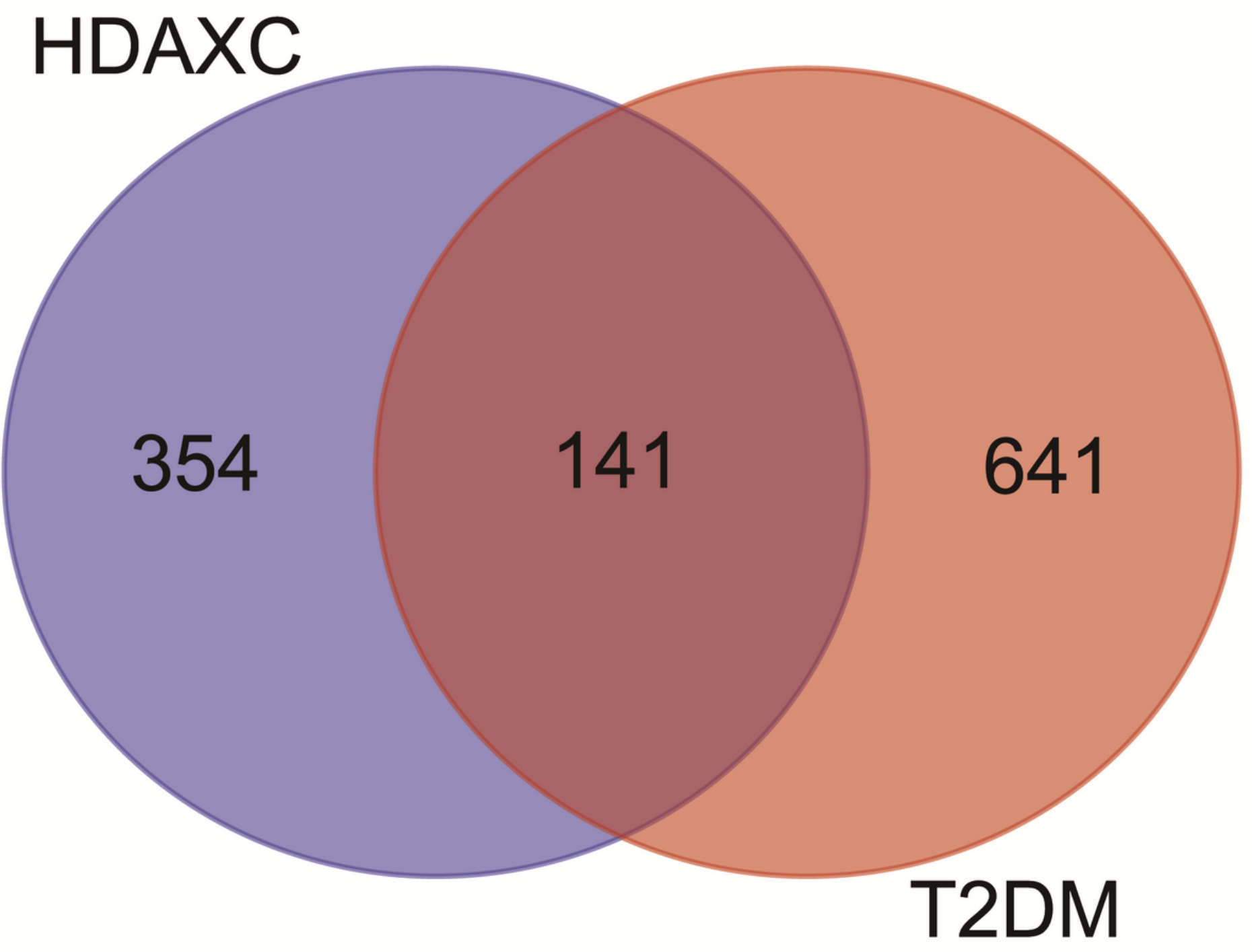

Figure 3

Matching of target genes between HDAXC and T2DM 


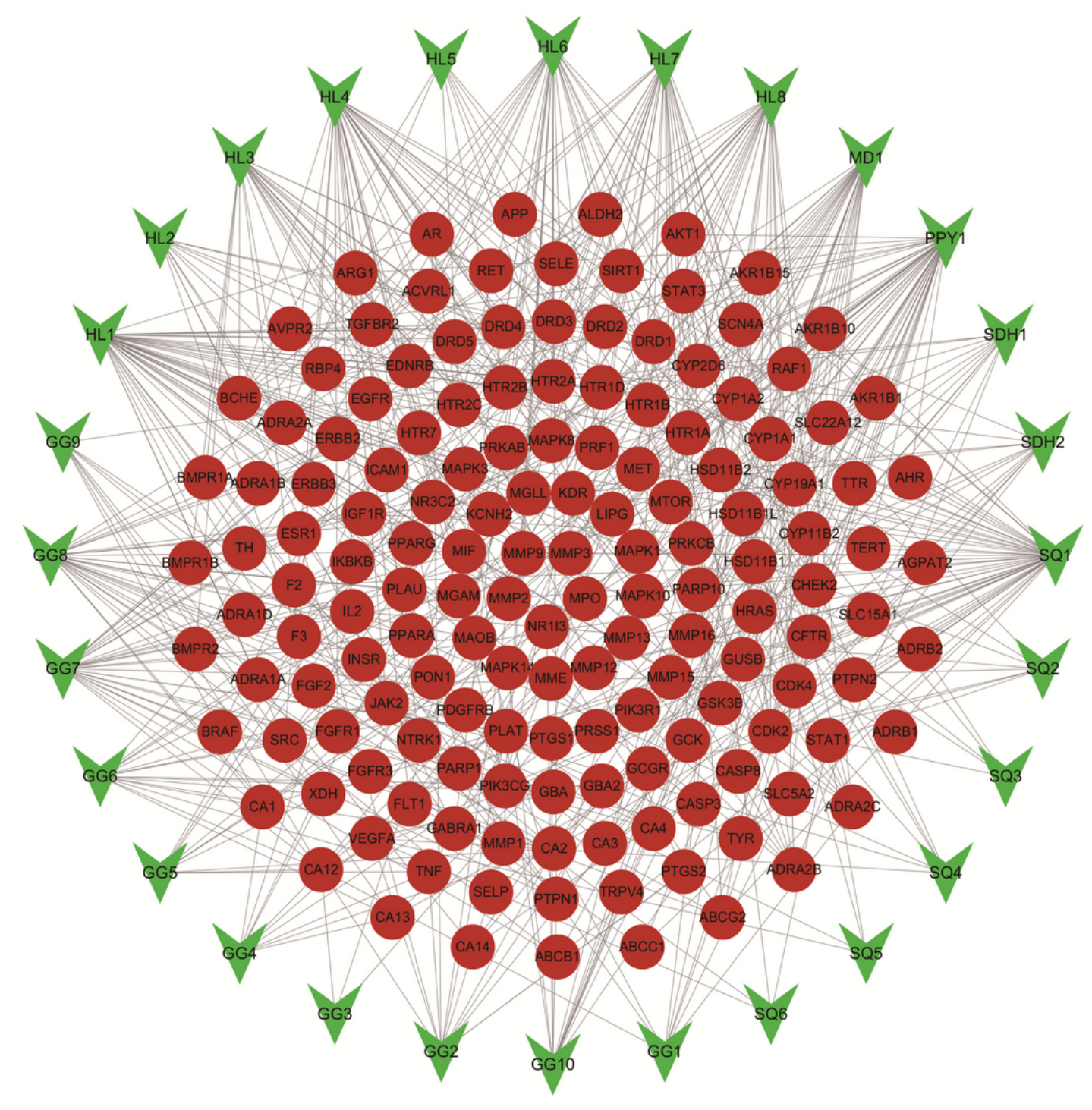

Figure 4

Compound-target network of potential targets in T2DM. The green nodes represent the potential active ingredients in T2DM, and the red nodes represent the corresponding targets of the ingredients and T2DM 


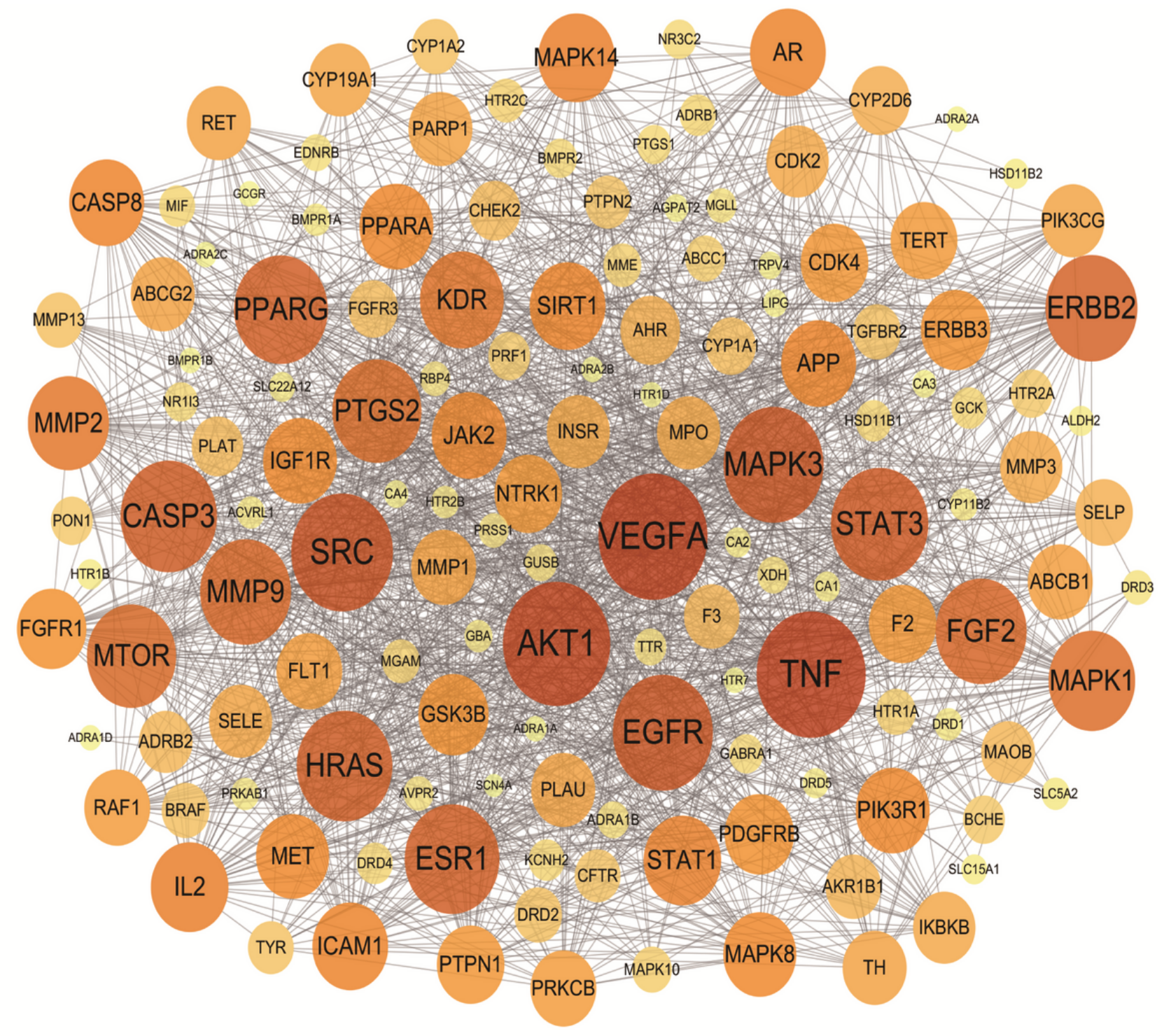

Figure 5

Common target PPI network between HDAXC and T2DM. The circles represent the common targets of the major active ingredients in the treatment of BC. The redder the color, the higher the degree 


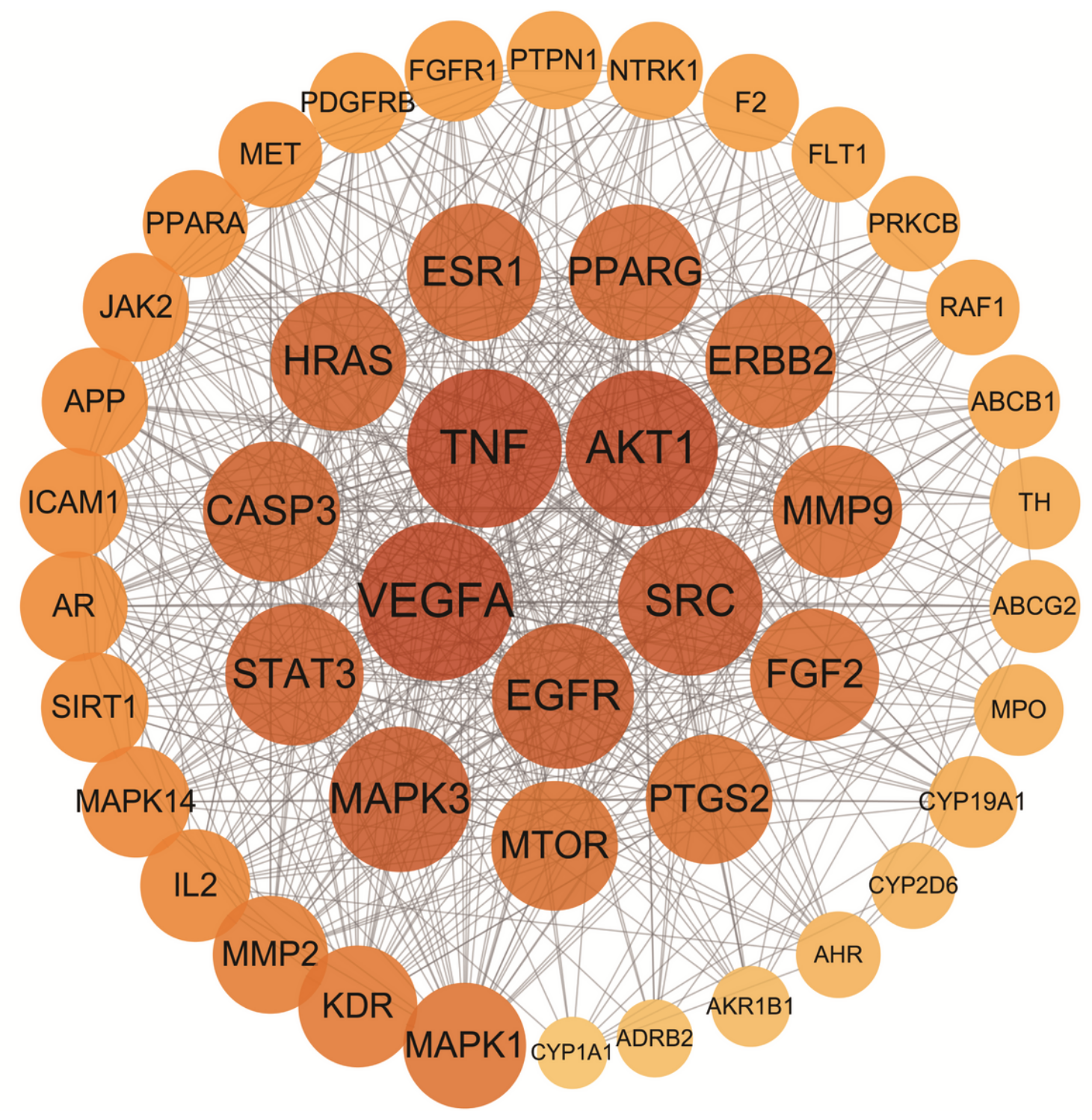

Figure 6

The core target of XHG treatment of BC in PPI network. The circles represent the common targets of the major active ingredients in the treatment of $\mathrm{BC}$. The redder the color, the higher the degree 


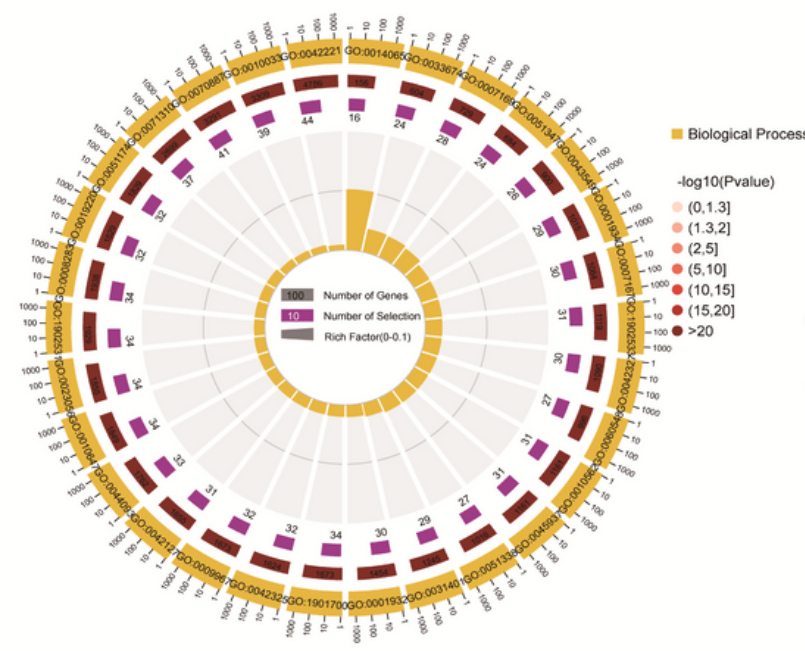

(a)

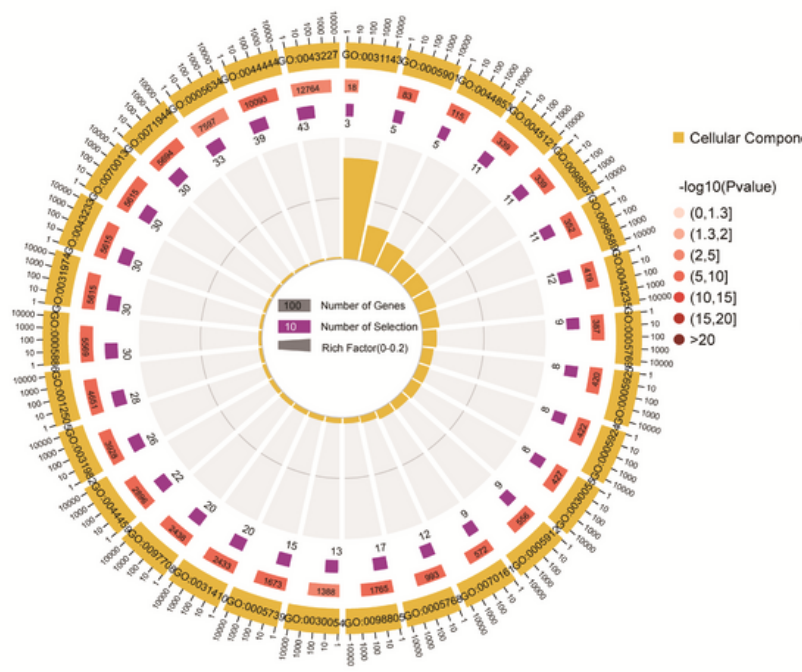

(C)

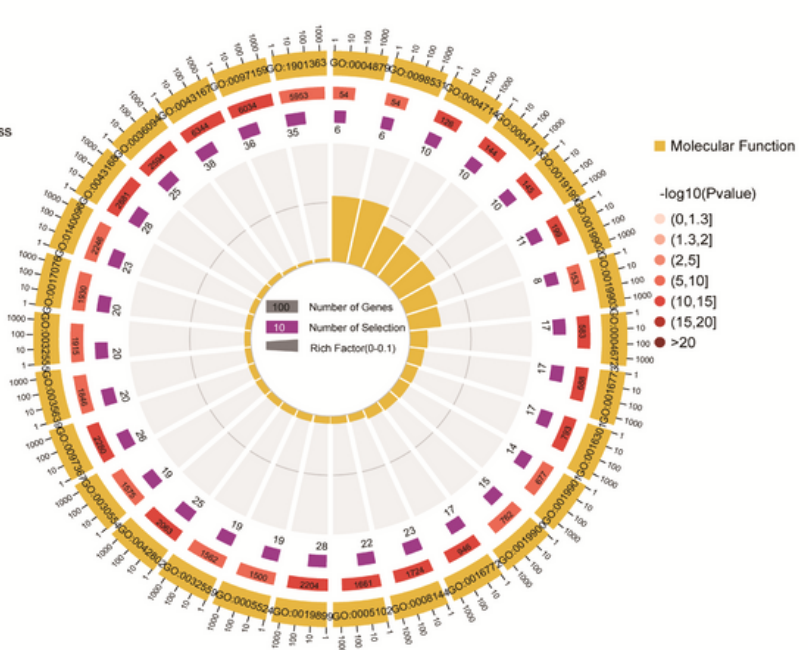

(b)

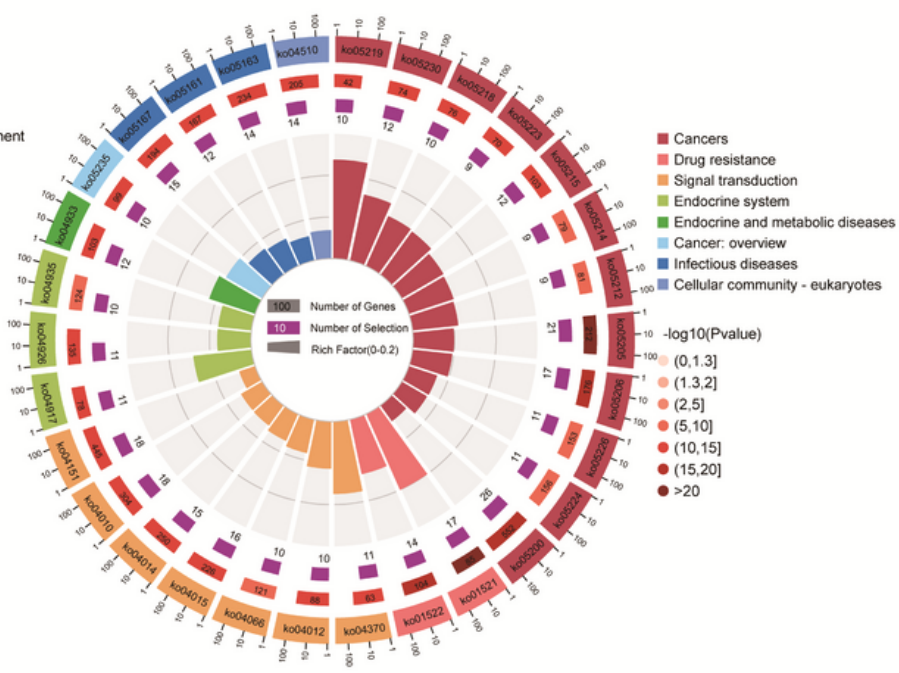

(d)

\section{Figure 7}

GO Enrichment and KEGG Pathway Analysis. (a) The first 30 enrichment BP analysis. (b) The analysis of the first 30 enrichment MF. (c) The first 30 enrichment CC analysis. (d) The KEGG pathway analysis of the first 30 significantly enriched 


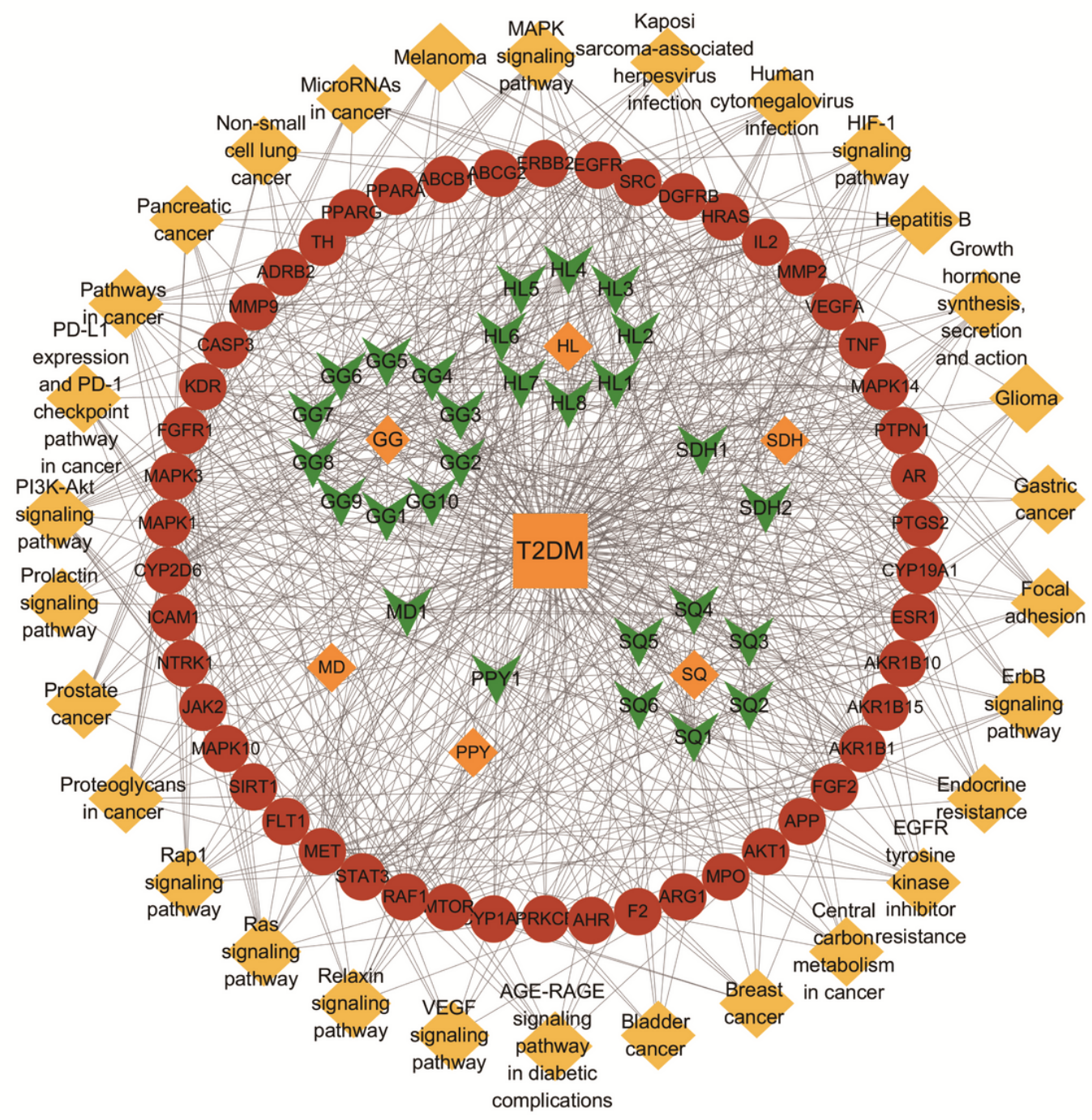

Figure 8

HDAXC-prescription composition-prototype components-targets-pathways". The orange square represents T2DM, the green v-shapes represent main drug compounds, the orange diamond represent the herbs of HDAXC, the red circular represent the intersection targets, and the yellow diamond represent the signal pathways 


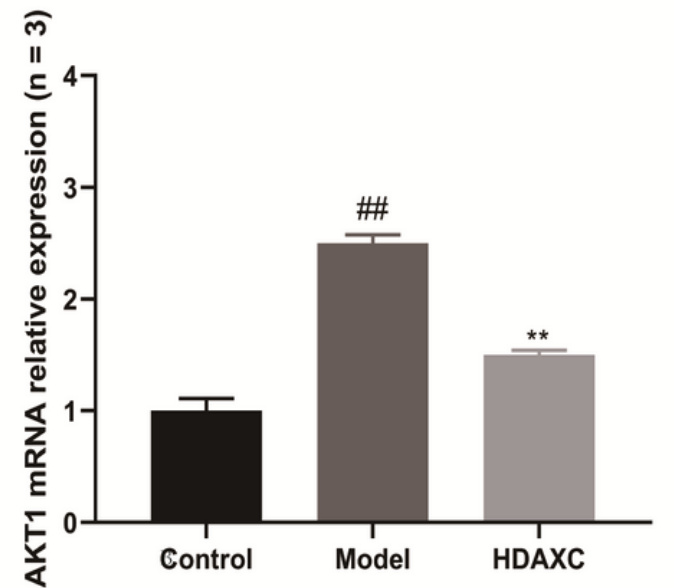

(a)

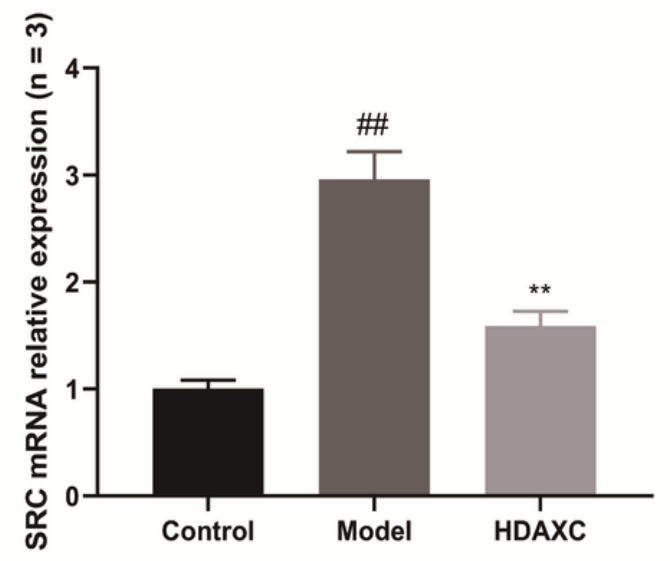

(c)

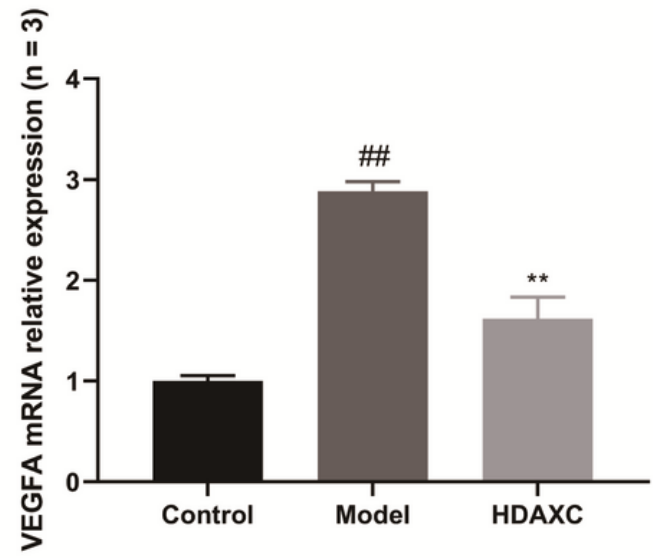

(b)

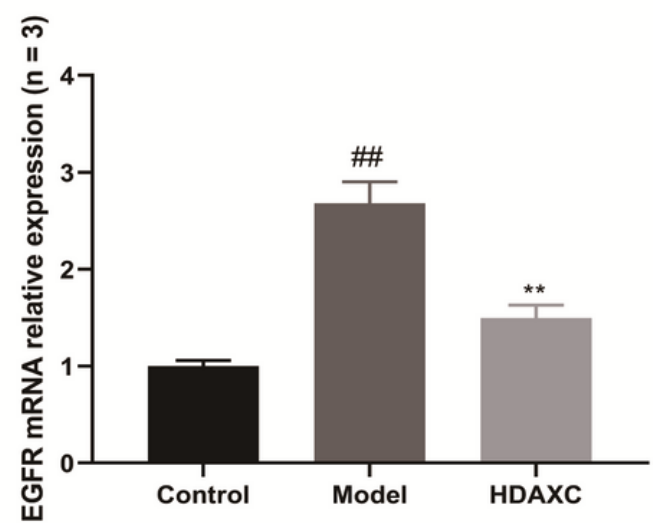

(d)

\section{Figure 9}

Change in AKT1, VEGFA, SRC, and EGFR mRNA expression was observed by qPCR. (a) Change in AKT1 mRNA expression was observed by qPCR. (b) Change in VEGFA mRNA expression was observed by qPCR. (c) Change in SRC mRNA expression was observed by qPCR. (d) Change in EGFR mRNA expression was observed by $\mathrm{qPCR}$. \#\#P<0.01 compared with the control group, $* * \mathrm{P}<0.01$ compared with the model group 


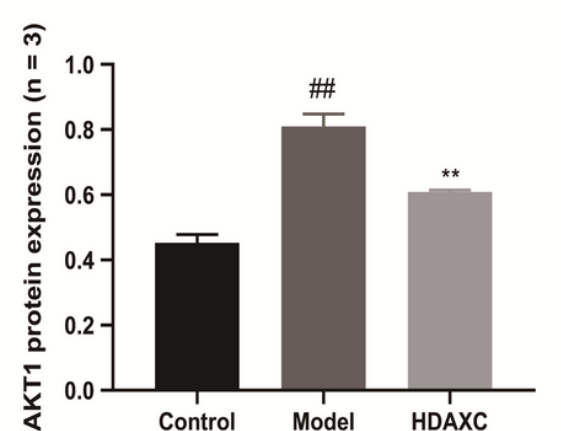

(a)

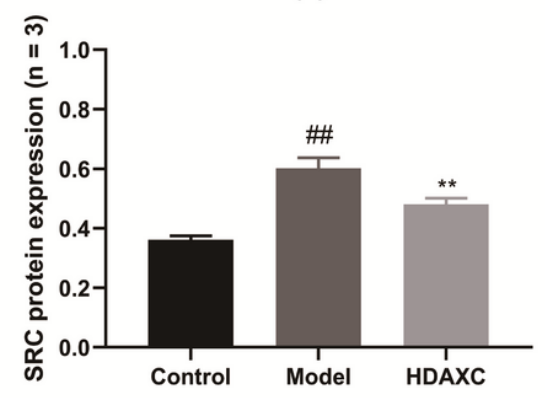

(c)

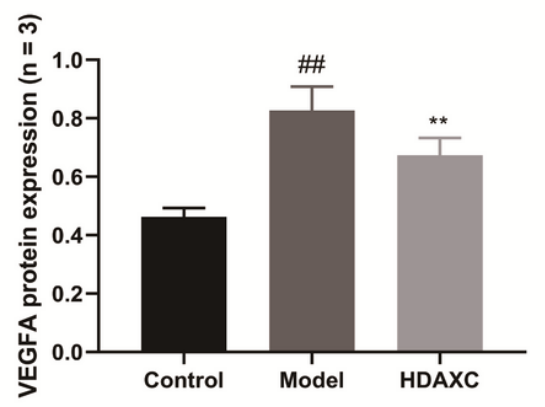

(b)

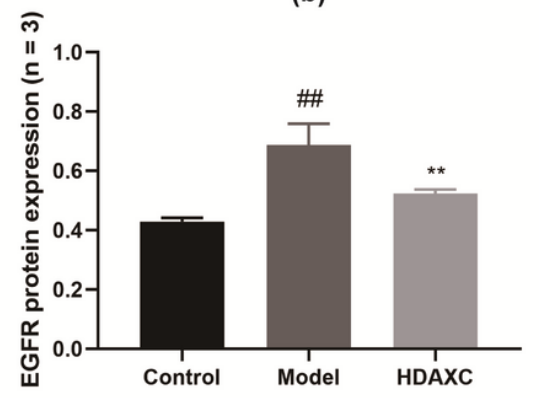

(d)

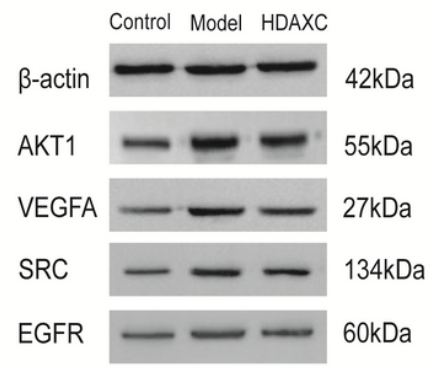

(e)

\section{Figure 10}

Change in AKT1, VEGFA, SRC, and EGFR protein expression was observed by Western bolt. (a) Change in AKT1 protein expression was observed by Western bolt. (b) Change in VEGFA protein expression was observed by Western bolt. (c) Change in SRC protein expression was observed by qPCR. (d) Change in EGFR protein expression was observed by Western bolt. (e) The protein levels were conducted by densitometric analysis of the blots following standardization to $\beta$-actin level. \#\#P<0.01 compared with the control group, $* * \mathrm{P}<0.01$ compared with the model group

\section{Supplementary Files}

This is a list of supplementary files associated with this preprint. Click to download.

- Supplementary1.xIsx

- Supplementary2.xlsx

- Supplementary3.xlsx

- Supplementary4.xlsx

- Supplementary5.xlsx

- Supplementary6.xlsx

- Supplementary7.tif 